\title{
Das unabhängige Belize und seine Verfassung
}

\author{
von Karl Leuteritz
}

I.

Am 21. September 1981 trat Belize - früher als Britisch Honduras bekannt - nach Art. 1 Abs. 1 seiner am Vortage verkündeten Verfassung1 als "souveräner demokratischer Staat Mittelamerikas in der karibischen Region" ins Leben. Mit der so umschriebenen Doppelzugehörigkeit zum sonst hispanophonen Mittelamerika einerseits und zur anglophonen Karibischen Gemeinschaft CARICOM andererseits wird sowohl das Spannungsverhältnis angesprochen, das die grundsätzlich längst beschlossene Unabhängigkeit des Landes fast zwei Jahrzehnte lang verzögerte, ${ }^{2}$ als auch die Brückenfunktion, die Belize nach Uberwindung dieser Spannungen bewußt für sich in Anspruch nimmt.

Die lange Wartezeit hat es dem belizischen Verfassungsgeber aber auch ermöglicht, bei seiner Arbeit die Erfahrungen anderer karibischer Commonwealth-Länder mit ihren Verfassungen zu verwerten, worauf der Bericht der vom 6. bis 14. April 1981 in London abgehaltenen Verfassungskonferenz besonders hinweist. ${ }^{3}$

So soll im folgenden versucht werden, zunächst die rechtliche Entstehungsgeschichte des belizischen Gemeinwesens abzuhandeln, die mit den teils bereinigten, teils fortbestehenden Territorialforderungen der Nachbarn Mexiko und Guatemala untrennbar verbunden ist. Dem folgt ein Abriß der belizischen Verfassung im Vergleich zu der als Modell besonders geeigneten Verfassung Jamaikas von 1962, während ein Blick auf das Verhältnis zwischen geschriebener Verfassung und politischer Wirklichkeit im unabhängigen Belize den Abschluß bildet.

II.

Die Darstellung der Entstehung eines eigenständigen belizischen Gemeinwesens und des britischen Souveränitätserwerbs über das heutige Staatsgebiet von Belize folgt im wesentlichen dem gemeinsamen Gutachten, das die britischen Völkerrechtler Elihu Lauter-

I A Constitution for an independent State of Belize (Belize Constitution); Act No. 14/1981 vom 20. 9. 1981. Zitiert als bel. Verf. Art. 1 Abs. 1 lautet: Belize shall be a sovereign democratic State of Central America in the Caribbean Region.

2 Vgl. Eröffnungsansprache des Staatsministers im britischen Außenministerium, Nicholas Ridley als Vorsitzender der Verfassungskonferenz in London am 6. 4. 1981: Report of the Belize Constitutional Conference, Marlborough House, London, 6-14 April 1981; Annex B. Government Printery, Belmopan 1981. Zitiert als Report.

3 Report a.a.O., § 10 
pacht und Derek W. Bowett 1978 der belizischen Regierung erstatteten. ${ }^{4}$ Dies ist die einzige greifbare Darstellung, die die komplexen Sachverhalte und Rechtspositionen in verständlicher Form zusammenfaßt. Dabei ist besonders hervorzuheben, daß der Gutachterauftrag nicht auf juristische Untermauerung einer bestimmten Position lautete, sondern auf Feststellung und Darlegung der Rechtslage, ${ }^{5}$ woran die Verfasser sich auch gehalten haben.

Danach lag die Souveränität über das Gebiet, wie über die benachbarten Regionen bis 1821 unbestritten bei der Krone Spaniens, wenn deren Rechte auch zunehmend durch vertragliche Konzessionen (Verträge von Paris 1763, Versailles 1783 und London 1786) zu Gunsten der seit dem 17. Jahrhundert an der Küste des "Golfs von Honduras" dem Holzeinschlag nachgehenden britischen Siedler ausgehöhlt wurden. Hinzu kamen das Entstehen einer rudimentären Selbstverwaltung mit einer Volksversammlung (Public Meeting) als Legislative und von dieser gewählten Friedensrichtern (Magistrates) als Exekutiv- und Rechtsprechungsorganen seit 1738 und die Entsendung eines dem Gouverneur von Jamaika unterstellten Aufsichtsbeamten (Superintendent) seit 1784. Demgegenüber beschränkte sich der spanische Souverän auf gelegentliche Inspektionen, um die Einhaltung der Vertragsbedingungen - keine landwirtschaftliche Nutzung, keine Befestigungen - zu überwachen (zuletzt 1796) und auf diplomatische Proteste gegen vertragswidrig doch errichtete Befestigungsanlagen an der Mündung des Belize-Flusses (zuletzt 1816). All diese Vorgänge aber bezogen sich ausschließlich auf das nördliche Drittel des heutigen Staatsgebietes zwischen den Flüssen Hondo im Norden und zunächst Belize, seit 1786 Sibun (etwa $20 \mathrm{~km}$ weiter südlich) im Süden. Auch gingen die spanischen Inspektionen und Proteste nicht etwa vom Generalkapitanat (Capitanía General) Guatemala aus, sondern von der Intendantur (Intendencia) Mérida de Yucatan, deren Verwaltungshoheit bis zum Belize- bzw. Sibun-Fluß reichte. ${ }^{6}$

Inzwischen hatten aber die britischen Siedler seit Beginn des 19. Jahrhunderts bei ihren Holzeinschlagsexpeditionen den Sibun nach Süden überschritten und trotz hinhaltenden Widerstands der aus London entsandten Superintendenten spätestens 1825 das Gebiet bis zum heutigen Grenzfluß Sarstoon faktisch okkupiert. Dieses Territorium gehörte theoretisch-kartografisch zum Generalkapitanat Guatemala, doch ist die Vornahme spanischer Hoheitsakte nicht feststellbar und auch von keiner Seite behauptet worden.?

Nachdem 1821 das Generalkapitanat Guatemala als "Zentralamerikanische Föderation“ seine Unabhängigkeit erklärt hatte und die Intendantur Mérida de Yucatan in dem

4 Belize; Joint Opinion by Mr. E. Lauterpacht, Q. C. and Dr. D. W. Bowett, Q. C.; Government Printery, Belmopan, o. J. Zitiert als Lauterpacht/ Bowett.

5 Lauterpacht/Bowett, a.a.O., $\S 1$ Satz 1: We have been asked to advise on the question of title to the territory of Belize (formerly British Honduras).

6 Lauterpacht/Bowett, a.a.O., §§ 12-23 und 36-44 mit den dort angeführten Vertragstexten und diplomatischen Dokumenten. Die in diesen Texten auftretende Schreibung "Wallis" bzw. "Walix" für den Belize-Fluß scheint übrigens die volksethymologische Deutung der geografischen Bezeichnung "Belize" als Verbalhornung des Namens des schottischen Seefahrers Wallace zu stützen, der im 17. Jahrhundert dort die erste Siedlung angelegt haben soll.

7 Lauterpacht/Bowett, a.a.O., $\S \S 20,45$. 
seit dem gleichen Jahr unabhängigen Mexiko aufgegangen war, erhoben beide Nachfolgestaaten Ansprüche auf Belize. Der mexikanische Anspruch beschränkte sich auf das alte Konzessionsgebiet zwischen Hondo und Sibun, während die Föderation und nach ihrem Auseinanderbrechen 1839 Guatemala das gesamte Gebiet zwischen Hondo und Sarstoon für sich beanspruchte, wobei sie sich auf eine angeblich 1787 vorgenommene Änderung der Verwaltungsgrenzen berief, die jedoch nie nachgewiesen werden konnte. Beide Staaten stützten ihre Ansprüche auf den seit 1810 als regionales Völkerrecht entwickelten Grundsatz "uti possidetis", nach dem die alten spanischen Verwaltungsgrenzen auch für die Abgrenzung der neu entstandenen Republiken maßgebend sein sollten. Großbritannien dagegen - und mit ihm die herrschende völkerrechtliche Lehrmeinung vertrat und vertritt die Auffassung, daß "uti possidetis" nur zwischen den Nachfolgestaaten Spaniens, nicht aber gegenüber Außenstehenden gelten könne und im übrigen ein Mindestmaß staatlicher Autoritätsausübung voraussetze, das weder Mexiko noch die Zentralamerikanische Föderation bzw. Guatemala je erbracht hätten. Als Spanien 1835 zur Anerkennung der Unabhängigkeit seiner ehemaligen lateinamerikanischen Kolonien bereit war, bemühte sich die britische Regierung deshalb in Madrid um die formelle Abtretung des Gebiets, jedoch ohne greifbares Ergebnis. ${ }^{8}$ Lauterpacht und Bowett kommen deshalb zu dem kaum zu widerlegenden Schluß, ${ }^{9}$ daß zu einem nicht genau bestimmbaren Zeitpunkt zwischen 1821 und 1850 die Souveränität über das Gebiet auf dem nicht eben häufigen Weg der völkerrechtlichen Ersitzung von Spanien auf Großbritannien übergegangen sei.

Die entgegenstehenden Ansprüche Mexikos wurden nach mehreren vergeblichen Anläufen schließlich durch den britisch-mexikanischen Vertrag vom 8. 7.1893 (ratifiziert 1897) ausgeräumt. ${ }^{10}$ Die scheinbar viel früher - nämlich durch den britisch-guatemaltekischen Grenzregelungsvertrag von 1859 (ratifiziert 12. 9. 1859) - beseitigten Ansprüche Guatemalas dagegen bestehen wegen der unterschiedlichen Interpretation dieses Vertrages bis heute fort. ${ }^{11}$ Entgegen dem klaren Wortlaut seines Art. 1 nämlich, der von der Festlegung der Grenze zwischen der Republik Guatemala und den "vor dem und am 1. 1.1850 bestehenden britischen Siedlungen und Besitzungen am Golf von Honduras" spricht, ${ }^{12}$ betrachtet Guatemala das Abkommen als Abtretungsvertrag, durch den Großbritannien die Souveränität über das Gebiet von Guatemala erworben habe. Die entgegenstehende Formulierung sei gewählt worden, um den auf Einhaltung der MonroeDoktrin bedachten USA keinen Grund zum Einschreiten zu bieten. Dieses Argument ist jedoch schon deswegen nicht stichhaltig, weil die "britische Siedlung in Honduras" von

8 Lauterpacht/Bowett, a.a.O., §§ 24-35, 55.

9 A.a.O., §§ 77-79.

10 Lauterpacht/Bowett, a.a.O., §§ 62-67.

11 Lauterpacht/Bowett, a.a.O.: Entstehungsgeschichte $\S \S 83-93$; Wortlaut $\S \S 94-96$, Interpretation $\S \S 97-116$.

12 Art. 1 lautet: "It is agreed between Her Britannic Majesty and the Republic of Guatemala that the boundary between the Republic and the British Settlement and Possessions in the Bay of Honduras, as they existed previous to and on the 1st day of January, 1850, and have continued to exist up to the present time, was, and is as follows: (folgt Beschreibung der Grenzlinie)“. Lauterpacht/Bowett, a.a.O., § 94. 
der Anwendung des britisch-amerikanischen Clayton-Bulwer-Vertrages vom 19. 4. 1850 - eines auf ganz Mittelamerika bezogenen Stillhalteabkommens - ausdrücklich ausgenommen worden war. ${ }^{13}$ Von entscheidender Bedeutung für die ganze Kontroverse ist aber Art. 7 des Vertrags van 1859, in dem sich Großbritannien und Guatemala in außerordentlich vagen Formulierungen verpflichten, sich gemeinsam zu bemühen, eine Verkehrsverbindung zwischen Guatemala-Stadt und der Atlantikküste bei Belize-Stadt herzustellen. ${ }^{14}$ Es ist unstreitig, daß diese Bestimmung nie ausgeführt worden ist und die britische Seite sich nicht sehr kooperativ gezeigt hat. Hieraus aber - wie Guatemala dies seit 1940 (!) tut - die Nichtigkeit des ganzen Vertrages abzuleiten und Belize als integrierenden Bestandteil des eigenen Staatsgebietes zu reklamieren, ist aus zwei Gründen auch dann rechtlich nicht haltbar, wenn man den guatemaltekischen Anspruch als vor $1859 \mathrm{zu}$ Recht bestehend anerkennen wollte: Einmal steht Art. 7 in keinem notwendigen inneren Zusammenhang mit dem übrigen Vertragsinhalt, so daß selbst seine schuldhafte Nichterfüllung diesen unberührt läßt. Zum anderen aber stellt der Positionswechsel Guatemalas angesichts seiner jahrzehntelangen Bemühungen - zuletzt noch 1938! -, Großbritannien zur Einhaltung seiner Verpflichtungen aus Art. 7 des Vertrages von 1859 zu bewegen, ein "venire contra factum proprium" dar, das nach dem in Art. 45 der Wiener Vertragsrechtskonvention kodifizierten allgemeinen Völkerrechtssatz unzulässig ist. ${ }^{15}$

So schwach also der guatemaltekische Anspruch rechtlich begründet ist, mit so großer Vehemenz wurde er immer wieder vorgebracht, noch 1977 mit Invasionsdrohung, die Großbritannien zu einer erheblichen Verstärkung seiner Garnison - auf etwa 1600 Mann - und zu ihrer Ausrüstung u. a. mit einigen Harrier Senkrechtstartern veranlaßte. Inzwischen war 1975 erstmalig von der Generalversammlung der VN unter Berufung auf das Selbstbestimmungsrecht in einer Resolution die baldige Entlassung Belizes in die Unabhängigkeit gefordert worden. Hatte es hier bei überwältigender Zustimmung (110 JaStimmen) noch 9 Nein-Stimmen (ausschließlich lateinamerikanische Staaten) und 16 Enthaltungen (darunter neben den übrigen lateinamerikanischen Staaten auch die USA) gegeben, so bröckelte diese Unterstützung Guatemalas von Jahr zu Jahr ab. ${ }^{16}$ Als schließlich 1980 auch die USA ins Lager der Befürworter überging, konnte die Resolution, da Guatemala sich nicht an der Abstimmung beteiligte, einstimmig verabschiedet

13 Wortlaut bei Lauterpacht/Bowett, a.a.O., $\S \S 69,70$.

14 Art. 7 lautet: "With the object of practically carrying out the views set forth in the preamble to the present Convention for improving and perpetuating the friendly relations which at present so happily exist between the two High Contracting Parties, they mutually agree conjointly to use their best efforts by taking adequate means for establishing the easiest communication (either by means of a cartroad, or employing the rivers, or both united, according to the opinion of the surveying engineers), between the fittest place on the Atlantic coast near the settlement of Belize and the capital of Guatemala; whereby the commerce of England on the one hand and the material prosperity of the Republic on the other, cannot fail to be sensibly increased, at the same time that the limits of the two countries being now clearly defined, all further encroachments by either party on the territory of the other will be effectually checked and prevented for the future." Lauterpacht/Bowett, a.a.O., § 96.

15 Lauterpacht/Bowett, a.a.O.: faktischer Ablauf $\S \S 117-143,154-157,179,180$; rechtliche Würdigung: $\S \S 114-116,144-153,158-178,180$ letzter Satz.

16 Vgl. Lauterpacht/Bowett, a.a.O., §§ 181-191. 
werden. Dies war um so beachtlicher, als erstmalig im Resolutionstext ein Termin (Ende 1981) für die Erlangung der Unabhängigkeit genannt wurde.

Offenbar unter dem Eindruck dieser Mahnung gewannen die sich seit Jahren ergebnislos hinschleppenden britisch-guatemaltekischen Verhandlungen neue Dynamik und führten am 11. 3. 1981 mit dem Abschluß einer Grundsatzvereinbarung (Heads of Agreement) zum Erfolg. In dieser 16 Punkte (heads) umfassenden Vereinbarung, ${ }^{17}$ an deren Aushandlung auf britischer Seite auch der Premier von Belize und zwei weitere belizische Minister beteiligt waren, verzichtete Guatemala überraschend auf seine Ansprüche und erkannte die territoriale Integrität Belizes an. Es begnügte sich mit Konzessionen hinsichtlich der Territorialgewässer - um die bei Inanspruchnahme von Zwölfmeilenzonen durch Belize und Honduras drohende Abschneidung seines Hafens Puerto Barrios vom Zugang zum offenen Meer zu verhindern - sowie mit der Einräumung von Freihafenund Transitrechten und von nicht näher definierten Nutzungsrechten (use and enjoyment) an den unbewohnten Inselgruppen der Ranguana- und Sapodilla Cayes. ${ }^{18}$

Auf dieser Grundlage wurde die Verfassungskonferenz vom 6. bis 14.4. 1981 durchgeführt, auf der Großbritannien und Belize nicht nur den Inhalt der neuen Verfassung für ein unabhängiges Belize vereinbarten, sondern sich auch über den einzuhaltenden Fahrplan verständigten. In den folgenden Wochen und Monaten gewann dann die Entwicklung eine derartige Eigendynamik, daß es bei der Entlassung Belizes in die Unabhängigkeit am 21. 9. 1981 blieb, obwohl Guatemala wegen Meinungsverschiedenheiten über die Natur der ihm an den Ranguana- und Sapodilla-Inseln einzuräumenden Nutzungsrechte $^{19}$ die zur vertraglichen Ausfüllung der Grundsatzeinigung inzwischen aufgenommenen Verhandlungen im August 1981 abgebrochen hatte. Der neue Staat wurde dann von der überwältigenden Mehrheit der Staatengemeinschaft einschließlich der übrigen mittelamerikanischen Staaten ${ }^{20}$ anerkannt und in die Vereinten Nationen aufgenommen. Guatemala freilich leugnet nicht nur die Existenz eines unabhängigen Belize, sondern hat auch die Grundsatzeinigung vom 11. 3. 1981 einseitig für nichtig erklärt. Úber neue vertragliche Regelungen will es nur mit Großbritannien verhandeln, das aber angesichts der neuen Rechtslage nur zu guten Diensten für etwaige guatemaltekisch-belizische Verhandlungen bereit ist. Seit Oktober 1982 sind in New York dreiseitige Gespräche unter Wahrung der jeweiligen Rechtsstandpunkte im Gange, d. h. Belize verhandelt unter Hinzuziehung britischer Vertreter mit Guatemala, das diese als eigentliche Partner ansieht und die belizische Delegation als Bestandteil der britischen betrachtet. Hinzu kommt die Aufrechterhaltung der britischen Sicherheitsgarantie und der Stationierung der britischen Garnison auf unbestimmte Zeit.

17 Text abgedruckt in: Government Explains Heads of Agreement. Government Printery, Belmopan, 1981.

18 Heads 1 (Anerkennung), 2 (Territorialgewässer), 4 (Freihafenrechte), 5 (Transitstraßen), 6 (Transit-Pipelines) und 3 (Nutzungsrechte an Inseln).

19 Belize und Großbritannien bestanden auf einer zeitlich begrenzten (längstens 99 Jahre) ausschließlich friedlichen Nutzung, während Guatemala jede zeitliche Begrenzung ablehnte und das Recht zur Errichtung eines Marinestützpunktes in Anspruch nahm.

20 Auch Honduras, das nach der Grundsatzeinigung vom 11. 3. 1981 überraschend eigene Ansprüche auf die Ranguana- und Sapodilla-Inseln erhoben hatte. 


\section{III.}

Bevor wir uns nun der belizischen Verfassung zuwenden, muß die innere Entwicklung des Landes seit dem frühen 19. Jahrhundert kurz nachgetragen werden: Nachdem schon 1840 durch Proklamation des Superintendenten englisches Recht als "Recht der Siedlung Britisch Honduras" eingeführt worden war, wurde das Gebiet auf wiederholte Petitionen der Siedler hin erst nach dem Grenzvertrag mit Guatemala schließlich 1862 durch königliches Patent zur Kronkolonie erklärt. ${ }^{21}$ Der dem Gouverneur von Jamaika unterstellte Superintendent wurde dabei durch einen unmittelbar dem Kolonialamt in London verantwortlichen Gouverneur abgelöst. Die Kolonie erhielt in den folgenden Jahrzehnten in mehreren Etappen innere Selbstverwaltung, bis schließlich eine als Úbergang zur Unabhängigkeit gedachte Verfassung von 1963 dem Gouverneur nur noch die Zuständigkeiten für Sicherheit und für Auswärtiges, daneben allerdings die Aufsicht über den öffentlichen Dienst beließ.22

Auf dieser Grundlage und auf den Erfahrungen, die andere karibische CommonwealthLänder mit ihren Verfassungen gemacht hatten, konnte die schon erwähnte Verfassungskonferenz vom April 1981 aufbauen. Das Ergebnis ihrer Arbeiten, eben die neue Verfassung von Belize, soll deshalb im Vergleich zur ältesten Verfassung eines karibischen Commonwealth-Staates, nämlich der Jamaikas von 1962, gewürdigt werden. Diese ist für einen solchen Vergleich auch deshalb besonders geeignet, weil Jamaika im Gegensatz etwa zu Guyana oder Trinidad und Tobago nicht zur republikanischen Staatsform übergegangen ist, sondern den Status einer Commonwealth-Monarchie in Realunion mit Großbritannien bewahrt hat, wie ihn auch die belizische Verfassung festlegt.

Hierbei fallen einige formale Unterschiede sofort ins Auge, während bei näherem Hinsehen inhaltlich eine weitgehende Ubereinstimmung festzustellen ist. So wurde die jamaikanische Verfassung - nach Aushandlung ihres Wortlauts auf der entsprechenden Verfassungskonferenz - als Anlage (schedule) einer britischen Staatsratsverordnung (Order in Council) verkündet und in Kraft gesetzt, ${ }^{23}$ während im Falle von Belize die Verfassungskonferenz sich mit Ausnahme der noch zu besprechenden Präambel auf die Ausarbeitung inhaltlicher Empfehlungen beschränkte und Formulierung und Beschlußfassung dem belizischen Parlament überließ. ${ }^{24}$ Hierin kommt der Emanzipationsprozeß zum Ausdruck, den auch die noch kolonial verwalteten britischen Territorien in den dazwischenliegenden 19 Jahren durchlaufen hatten. Besonderen Ausdruck findet dieses gesteigerte Selbstbewußtsein auch in der Präambel, die in sechs prägnanten Absätzen feststellt, daß das Volk von Belize

21 Lauterpacht/Bowett, a.a.O., §§ 49-52.

22 Report, a.a.O., Annex B.

23 Statutory Instruments 1962 No. 1550 vom 23. 7. 1962: The Jamaica (Constitution) Order in Council 1962; Second Schedule: The Constitution of Jamaica. Zitiert als jam. Verf.

24 Report, a.a.O., §§ 6-107; vgl. auch oben Anm. 1. 
- den Willen bestätigt, die Nation auf dem Anerkenntnis göttlicher Allmacht und dem Glauben an die Menschenrechte und Grundfreiheiten zu begründen;

- den Grundsatz sozialer Gerechtigkeit mit den sich daraus ergebenden politischen Folgen respektiert;

- daran glaubt, daß der in freien Wahlen zu ermittelnde Volkswille die Grundlage einer demokratischen Gesellschaft bildet;

- den Rechtsstaat (rule of law) als Garanten freiheitlicher Institutionen anerkennt;

- eine Politik fordert, die die Einheit, Freiheit und territoriale Integrität Belizes schützt, die Chancengleichheit verwirklicht, die Individualrechte einschließlich des Rechts auf Eigentumserwerb und selbständige wirtschaftliche Betätigung schützt, dabei aber die Ausbeutung des Menschen durch den Menschen oder durch den Staat verbietet und die schließlich die Errichtung einer gerechten Friedensordnung nach innen und außen zum Ziel hat; und

- die Verankerung dieser Grundsätze und Ziele in seiner Verfassung wünscht. ${ }^{25}$

Die jamaikanische Verfassung dagegen hat keine Präambel und beginnt wie viele britische Gesetze sehr prosaisch mit einer langen Aufzählung von Legaldefinitionen, die in der belizischen Verfassung ganz ans Ende der operativen Bestimmungen gerückt wurden. ${ }^{26}$ Schließlich sind in Belize die Übergangs- und Schlußvorschriften Bestandteil der Verfassung selbst, während sie für Jamaika in der Mantelverordnung des britischen Staatsrats enthalten sind. ${ }^{27}$

Inhaltlich regelt die Verfassung in zwölf Kapiteln (142 Artikeln) "Staat und Verfassung" (I), "Schutz der Grundrechte und -freiheiten" (II), "Staatsangehörigkeit" (III), "Generalgouverneur" (IV), "Exekutive" (V), "Legislative" (VI), "Rechtsprechende Gewalt" (VII), "Offentlicher Dienst" (VIII), "Finanzen" (IX), "Verschiedenes" (X), "Ưbergangsbestimmungen" (XI) und "Aufhebung und Inkrafttreten" (XII). Im Vergleich zu der entsprechend dem Fehlen von Ubergangs- und Schlußvorschriften nur zehn Kapitel umfassenden jamaikanischen Verfassung ${ }^{28}$ ist die Bedeutung der Grund- und Freiheitsrechte neben der Erwähnung in der Präambel auch dadurch stärker betont, daß das entsprechende Kapitel gleich auf die grundlegenden Bestimmungen über Staat und Verfassung folgt, während dort die Staatsangehörigkeitsbestimmungen dazwischengeschaltet sind. Auch wird die Exekutive im weiteren Sinne in Kap. IV und V (Art. 30-54) zusammenhängend behandelt, wofür man allerdings in Kauf nahm, daß die Bestimmungen über die Legislative, aus der die Regierung als Exekutive im engeren Sinne ja hervor-

25 Der von der Verfassungskonferenz erarbeitete Wortlaut - Report $\S 6$ und Annex D - wurde unverändert übernommen.

26 Art. 1 jam. Verf., Art. 131 bel. Verf.

27 Art. 132-142 bel. Verf.; Art. 3-20 Staatsrats-VO, a.a.O. Die jamaikanische Lösung orientiert sich dabei an der vor der Entlassung in die Unabhängigkeit bestehenden Rechtslage, die belizische an der politischen Wirklichkeit.

28 Diese behandeln: "Einleitung" (I), "Staatsangehörigkeit" (II), "Grundrechte und -freiheiten" (III), "Generalgouverneur" (IV), "Parlament) (V), "Exekutivgewalt" (VI), "Rechtsprechende Gewalt" (VII), "Finanzen" (VIII), "Offentlicher Dienst" (IX) und "Verschiedenes" (X). 
geht, sich unlogischerweise erst im folgenden Kap. VI (Art. 55-93) finden. Ob diese von der Verfassungskonferenz empfohlene Abweichung vom jamaikanischen Vorbild, wo die Legislative zwischen den Bestimmungen über den Generalgouverneur und über die Exekutive behandelt wird ${ }^{29}$ wirklich ein Fortschritt ist, sei dahingestellt. Dies gilt erst recht für die Umstellung der Reihenfolge zwischen den beiden Kapiteln über den öffentlichen Dienst und Finanzen. ${ }^{30}$

Im einzelnen enthält das nur aus zwei Artikeln bestehende Kap. I in Art. 1 neben der zu Beginn dieses Aufsatzes zitierten programmatischen Staatsdefinition als Abs. 2 noch einen Verweis auf die als Anhang (schedule) 1 beigefügte geografische Beschreibung des Staatsgebiets. Art. 2 stipuliert dann den Vorrang der Verfassung vor dem übrigen Landesrecht, wobei die Formulierung zwar grundsätzlicher klingt als in der entsprechenden jamaikanischen Vorschrift, inhaltlich aber nicht über sie hinausgeht. ${ }^{31}$ Auffälligerweise fehlt aber die von der Verfassungskonferenz an dieser Stelle empfohlene Definition der Staatsform als konstitutionelle Monarchie mit der (britischen) Königin als durch einen Generalgouverneur vertretenes Staatsoberhaupt. ${ }^{32}$ Diese muß vielmehr aus anderswo verstreuten Vorschriften - Bestellung des Generalgouverneurs durch die Königin Art. 30; die Königin als Inhaberin der Exekutive gar erst Art. 36; Bildung der Regierung durch die Parlamentsmehrheit Art. 37 Abs. 2 - erschlossen werden. ${ }^{33}$

Das folgende Grundrechtskapitel (Art. 3-22) garantiert entsprechend den in der Präambel postulierten Grundsätzen zunächst in Art. 3 jeder in Belize befindlichen Person ohne Rücksicht auf Rasse, Herkunft, politische Meinung, Hautfarbe, Glauben oder Geschlecht, aber vorbehaltlich der Achtung der Rechte und Freiheiten anderer und des öffentlichen Interesses die folgenden Rechte und Freiheiten:

- Recht auf Leben, Freiheit, Sicherheit der Person und rechtlichen Schutz;

- Freiheit des Gewissens, des mündlichen und schriftlichen Ausdrucks, der Versammlung und Organisation;

- Schutz der Familie, der persönlichen Privatsphäre und der Wahrung und Anerkennung ihrer Menschenwürde, sowie

- Schutz vor willkürlicher Enteignung.

In den Artikeln 4-17 werden diese Garantien spezifiziert, wobei auf das Verbot entwürdigender Behandlung (Art. 7) und das historisch zu erklärende Verbot der Sklaverei (Art. 8 Abs. 1) besonders hingewiesen sei. In dem die Versammlungs- und Organisationsfreiheit regelnden Art. 13 wird das Recht auf die Bildung von Gewerkschaften und politischen Parteien hervorgehoben. Auch das in Art. 15 postulierte Recht auf Arbeit und freie Berufswahl verdient Beachtung. Wo einzelne Grundrechte unter Gesetzesvor-

29 Report, a.a.O., §§ 24-26 (Generalgouverneur), 27-45 (Exekutive), 46-78 (Legislative).

30 Offentlicher Dienst Art. 105-113 bel. Verf., 123-134 jam. Verf.; Finanzen Art. 114-120 bel. Verf., 114-122 jam. Verf.; vgl. Report, a.a.O., §§ 90-98 (öffentlicher Dienst), 99-105 (Finanzen).

31 Vgl. Art. 2 jam. Verf.

32 Report, a.a.O., § 8.

33 Dem entsprechen Art. 27, 68 (!) und 70 Abs. 1 jam. Verf. 
behalt stehen, wie die Eigentumsgarantie in Art. 17, wird für Eingriffsgesetze ein Minimalstandard festgelegt, hier z. B. angemessene Entschädigung und Möglichkeit der gerichtlichen Anfechtung. Art. 18 und 19 lassen im Falle eines entweder vom Generalgouverneur proklamierten oder durch Resolution des Parlaments festgestellten Notstandes stärkere Eingriffe zu, enthalten aber auch für diesen Fall umfangreiche Schutz- und Rechtsmittelvorschriften für die Betroffenen. Art. 20 hält für alle Grundrechtsverletzungen den Rechtsweg einschließlich der Revisionsmöglichkeit an den britischen Staatsrat (Judicial Committee of the Privy Council) offen, während Art. 21 für nicht grundrechtkonformes altes Recht eine Schonfrist von fünf Jahren gewährt und Art. 22 einige Legaldefinitionen enthält. Viele dieser Bestimmungen stimmen fast wörtlich mit den entsprechenden Vorschriften der jamaikanischen Verfassung überein, ${ }^{34}$ doch ist in einigen Fällen die Schutzfunktion stärker betont und erweitert. ${ }^{35}$ Auch ist das Recht auf Arbeit und freie Berufswahl in der jamaikanischen Verfassung noch nicht ausdrücklich erwähnt.

Die Staatsangehörigkeitsbestimmungen in Art. 23-29 legen gemäß dem im angelsächsischen Rechtskreis vorherrschenden "ius soli" die Geburt in Belize als Regelfall des Staatsangehörigkeitserwerbs fest (Art. 23, 24), doch erwerben gemäß Art. 25 auch im Ausland geborene Kinder belizischer Eltern die heimatliche Staatsangehörigkeit durch Geburt. Die übrigen Bestimmungen dieses Kapitels regeln neben dem Staatsangehörigkeitserwerb durch Heirat und durch Einbürgerung auch die Verlusttatbestände. Materiell gleichen diese Vorschriften den jamaikanischen, doch ist die dort noch praktizierte Privilegierung von Staatsangehörigen "anderer" Commonwealth-Staaten (außer Großbritannien) weggefallen. ${ }^{36}$

In den drei Kapiteln über die obersten Staatsorgane - Generalgouverneur Art. 30-35, Exekutive Art. 36-54, Legislative Art. 55-93 - wird Belize als parlamentarische Monarchie nach dem "Westminster-Modell" organisiert, ohne daß dies, wie schon erwähnt, ausgesprochen wird: Der die Königin als Inhaberin der Exekutive vertretende und von ihr ernannte Generalgouverneur, der Belizer sein muß, ${ }^{37}$ beruft zum Ministerpräsiden$\operatorname{ten}^{38}$ den Führer der Mehrheitspartei im Repräsentantenhaus, während der Führer der Minderheit zum Oppositionsführer berufen wird, den die Verfassung ebenfalls zur Exekutive rechnet. Auf Vorschlag des Ministerpräsidenten ernennt der Generalgouverneur dann die Ressortminister, die dem Parlament angehören müssen und gemeinsam das Kabinett bilden. ${ }^{39}$ Für den Justizminister - Attorney General - wird eine fünfjährige ju-

34 Art. 13-26 jam. Verf.

35 So spricht Art. 3 bel. Verf. vom Schutz der Familie usw., Art. 13 jam. Verf. nur von ihrer Achtung. In den Schutz der Organisationsfreiheit sind in Jamaika politische Parteien noch nicht eingeschlossen.

36 Art. 3-12 jam. Verf.; Commonwealth-Privilegien, Art. 9, 10.

37 Dieses Qualifikationsmerkmal des Art. 30 bel. Verf. fehlt in dem entsprechenden Art. 27 jam. Verf.

38 Diese Bezeichnung erscheint dem Verfasser besser als das im Deutschen übliche "Premierminister" geeignet, den "Prime Minister" eines unabhängigen Staates vom "Premier" einer Kolonie mit Selbstregierung abzuheben.

39 Diese Reihenfolge in Art. 37, 40, 44 bel. Verf. entspricht eher der politischen Wirklichkeit als die umgekehrte in Art. 69-71 jam. Verf.; die das Kabinett an die Spitze stellt. 
ristische Berufserfahrung vorgeschrieben. Schließlich steht dem Generalgouverneur ein Beirat (Belize Advisory Council) von mindestens sechs Mitgliedern zur Seite, die er auf Vorschlag des Ministerpräsidenten beruft, der dafür bei zweien des Einvernehmens des Oppositionsführers bedarf. ${ }^{40}$ Die Legislative besteht aus zwei Kammern: Dem Repräsentantenhaus von gegenwärtig 18 in Einmannwahlkreisen nach relativem Mehrheitswahlrecht gewählten Abgeordneten und dem aus acht ernannten Senatoren bestehenden Senat. Wählen diese ein Nichtmitglied zum Präsidenten, so tritt dieser als neunter Senator hinzu. Für die Ernennung der übrigen acht ist für fünf der Vorschlag des Ministerpräsidenten maßgebend, für zwei der des Oppositionsführers. Den achten ernennt der Generalgouverneur nach Anhörung des Beirats nach freiem Ermessen. Neben kleineren formalen Abweichungen ist hier auf zwei materielle Unterschiede zu Jamaika hinzuweisen, von denen der erste mehr staatstheoretische, der zweite politische Bedeutung hat: Die Königin ist nicht mehr Bestandteil auch der Legislative, und neu ist der "neutrale" Senator: Alle 21 jamaikanischen Senatoren werden im Verhältnis 13 zu 8 auf Vorschlag des Ministerpräsidenten und des Oppositionsführers ernannt. ${ }^{41}$ Mit Ausnahme von haushaltsrelevanten Gesetzen (money bills) hat der Senat gegenüber den Beschlüssen des Repräsentantenhauses ein aufschiebendes Veto.

In den die Rechtsprechung regelnden Art. 94-104 wird die Unabhängigkeit der Richter und Gerichte garantiert und ein zweistufiger Gerichtsaufbau mit Oberstem Gericht (Supreme Court) und Berufungsgericht (Court of Appeal) errichtet. Als dritte Instanz fungiert "Her Majesty in Council", d. h. der Rechtsausschuß des britischen Staatsrats in London.

Hauptzweck der den öffentlichen Dienst behandelnden Art. 105-113 ist es, durch die Errichtung eines aus einem Vorsitzenden und 12 Mitgliedern bestehenden Personalausschusses (Public Services Commission), dem alle Entscheidungen über Ernennungen und Beförderungen und Disziplinarmaßnahmen wie auch die Besoldungsregelung vorbehalten sind, parteipolitische Einflußnahmen zurückzudrängen. Von den 12 ordentlichen Mitgliedern sind je vier für den allgemeinen öffentlichen Dienst und für das Militär, je zwei für die Justiz und für die Polizei zuständig.

Das Finanzkapitel - Art. 114-120 enthält haushaltsrechtliche Grundsatzbestimmungen und sieht einen an Weisungen nicht gebundenen "Auditor General" als Rechnungsprüfungsinstanz vor. Von den unter der Uberschrift "Verschiedenes" zusammengefaßten Vorschriften der Art. 121-131 sei hier nur auf den Verhaltenskodex für Amtsträger hingewiesen, die keinen Disziplinarvorschriften unterliegen (Art. 121): Er ist vor allem auf die Vermeidung von Interessenkonflikten gerichtet und hat keine jamaikanische Ent-

40 Die Mitglieder des entsprechenden jamaikanischen Gremiums, das noch nach britischem Vorbild Staatsrat (Privy Council) heißt, werden gemäß Art. 82 jam. Verf. vom Generalgouverneur nach Anhörung des Ministerpräsidenten ernannt.

41 Nach Art. 55 bel. Verf. besteht die Nationalversammlung aus Repräsentantenhaus und Senat, nach Art. 34 jam. Verf. das Parlament aus Ihrer Majestät, dem Senat und dem Repräsentantenhaus; Ernennung der Senatoren: Art. 61 bel. Verf., Art. 35 jam. Verf. 
sprechung. Alle anderen Bestimmungen dieses wie der vorangehenden Kapitel stimmen inhaltlich mit jamaikanischen Vorschriften überein. ${ }^{42}$ Die Úbergangs- (Art. 132-140) und Schlußbestimmungen (Art. 141-142) beschränken sich auf das Notwendige und Ubliche. Bemerkenswert ist, daß das aus den letzten Wahlen vor der Unabhängigkeit (1979) hervorgegangene Parlament und die von diesem gebildete Regierung bis zu den turnusmäßig 1985 fälligen Neuwahlen mit den in der neuen Verfassung vorgesehenen Befugnissen im Amt bleiben, wie dies s. Z. auch in Jamaika der Fall war. ${ }^{43}$

IV.

So steht die belizische Verfassung als ein Bau vor uns, der zunächst einmal auf dem Papier allen Anforderungen an einen demokratisch-parlamentarischen Rechtsstaat genügt. Aber auch die politische Wirklichkeit entspricht diesem staatsrechtlichen Entwurf viel mehr, als Skeptiker dies in einem Entwicklungsland mit heterogener Bevölkerungsstruktur erwarten mögen: Der potentiell gefährliche Gegensatz zwischen der anglophonen Bevölkerungsmehrheit von 60-70\% der etwa 145000 Einwohner und der hispanophonen Minderheit von 30-40\%, wozu noch Splitter-Minderheiten wie reine Maya Indianer, schwarze Kariben und deutschstämmige Mennoniten kommen, wird in den beiden großen Parteien integriert, die sich nach der Gewährung der vollen inneren Selbstregierung 1963 herausgebildet haben: Dem Kabinett des Ministerpräsidenten Price gehören anglound hispanophone Minister an und Price selbst hält alle seine Reden englisch und spanisch; der bisherige Oppositionsführer Dr. Aranda (im Dezember 1982 zurückgetreten) ist Karibe, sein Vertreter Esq̀uivel hispanophoner Mestize. Da zudem fast alle hispanophonen Belizer oder ihre Vorfahren vor diktatorischen Regimen in einem der benachbarten Länder nach Belize geflohen sind, stellen sie dessen demokratische Strukturen nicht in Frage. Einem Umkippen der Mehrheitsverhältnisse durch das Hereinströmen von Flüchtlingen aus El Salvador sucht man durch ein Abkommen mit dem Hohen Flüchtlingskommissar der Vereinten Nationen über die Hereinnahme illegaler haitianischer Einwanderer aus den USA entgegenzuwirken: Sie dürften sich eher dem rassisch verwandten anglophonen Bevölkerungsteil assimilieren als dem hispanophonen.

Von den beiden großen, allein im Parlament vertretenen Parteien stellt die eher sozialdemokratische PUP (Peoples' United Party) seit 1964 den Regierungschef Price. Dieser hat es auch verstanden, prokubanische Tendenzen des linken PUP-Flügels klug zu integrieren und damit zu neutralisieren. Die eher liberale UDP (United Democratic Party) aber ist nicht so unbedeutend, wie es ihre nur fünf Sitze (von 18) im Repräsentantenhaus vermuten lassen könnten. Auf kommunaler Ebene hatte sie mehrfach - auch in BelizeStadt - beachtliche Erfolge und konnte so administrative Erfahrungen sammeln. 
Die Auseinandersetzung zwischen Regierung und Opposition hat sich ganz überwiegend in demokratischer Form abgespielt. Nur der künstlich hochgespielte Konflikt um "sofortige Unabhängigkeit" (PUP) oder "Unabhängigkeit erst nach vertraglicher Einigung Großbritanniens mit Guatemala" (UDP) führte im April 1981 zu Gewalttätigkeiten. Nachdem es Price aber wider Erwarten gelungen war, die britische Sicherheitsgarantie auch nach der Unabhängigkeitserklärung zu erlangen und er der UDP in einigen psychologisch wichtigen Punkten - zwei schmale rote Streifen in der blau-weißen Nationalflagge, freier Zugang zu den Medien, bessere Wahrung der Chancengleichheit bei künftigen Wahlen - entgegengekommen war, nimmt die Opposition ihre Rolle wieder loyal wahr. So kann man dem neuen Staatswesen trotz der latent fortbestehenden Bedrohung durch Guatemala politisch durchaus eine günstige Prognose stellen. 


\section{Misunderstandings over India. British Colonial Government and present structure pro- blems}

\section{By Ingeborg $Y$. Wendt}

It is still customary to trace back India's poverty to "traditionalism", illiteracy, and overpopulation, or a high rate of population growth. But these concepts are vague in various respects. Illiteracy was widespread in 19th century Europe when industrialization began, and it disappeared only gradually, along with technical progress, i. e., wide-spread literacy was not a pre-condition to industrialization, but a result. As for the growth of population: the Japanese population grew between the 1860s and 1929, the period of the start and the main phase of Japanese industrialization, from 30 to more than 60 millions. This growth did, obviously, not impede progress. Today's India may be the most densely populated country of the South, but as such it equals only northern France with respect to the population / arable land ratio, and France is far less densely populated than West Germany. The most controversial item is the Western projection of Indian "traditionalism". It pretends to refer to the ancient Indian past, and it ignores the European colonial reign of several centuries which form a tradition closer and more relevant to the present times. During the last centuries India's native social structures were broken by foreign power, and a foreign structure was super-imposed as it was in most other countries outside the West.

It is this still prevailing foreign sur-structure which is responsible for India's poverty, as for the poverty of the South in general.

\section{Independent Belize and its Constitution}

\section{By Karl Leuteritz}

The small colonial possession of British Honduras gained independence on 21 st September 1981, as the state of Belize. This article outlines the historical and diplomatic developments, from the late eighteenth to the late nineteenth century, which transformed the territory from a Spanish dependency inhabited by numerous British settlers into a Crown Colony. The recent initiatives in the United Nations in preparation of independence are briefly described, together with the political and legal issues persisting between Guatemala and the new country. The main part of the article presents the characteristic features of the Belize constitution of 1981, with particular reference to the 1962 Jamaican constitution which, unlike some other constitutions of former British colonies in the Caribbean region, also preserved the country's institutional links with Great Britain. A short survey of contemporary Belize politics concludes the presentation. 